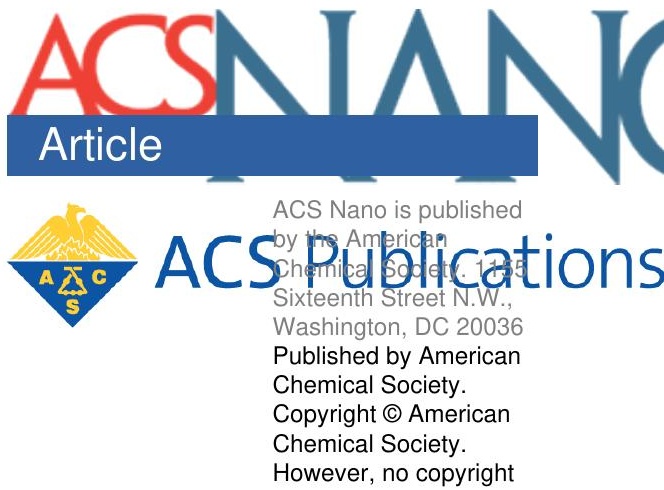




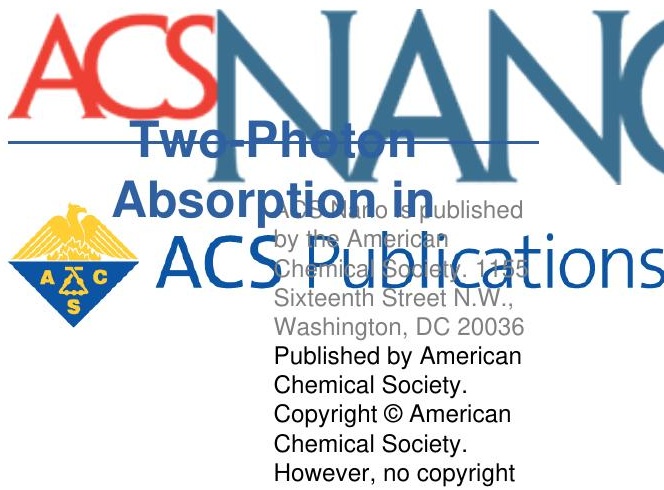




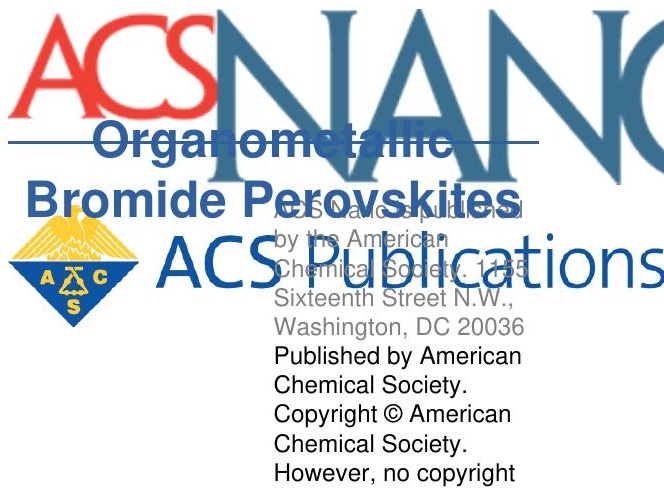




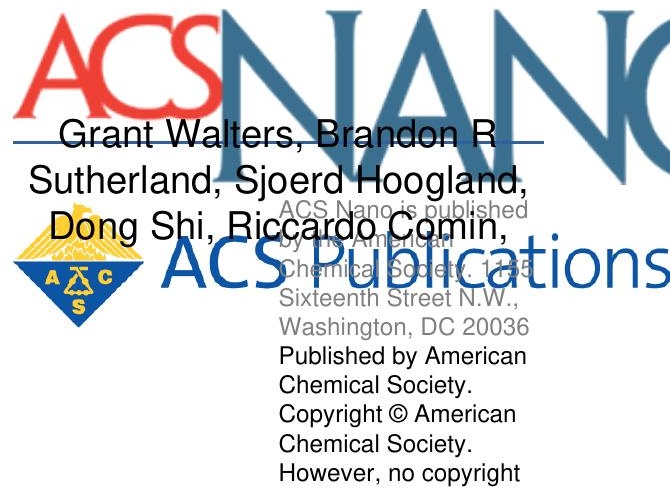



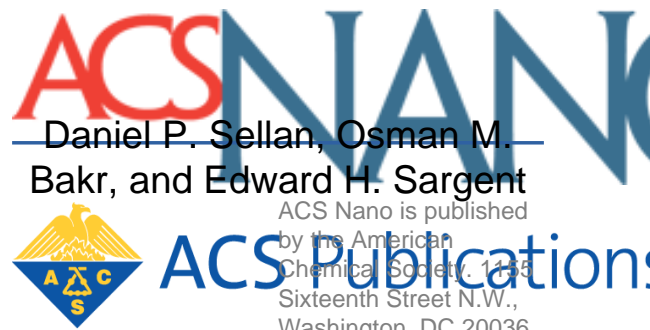

ACS Nano is published

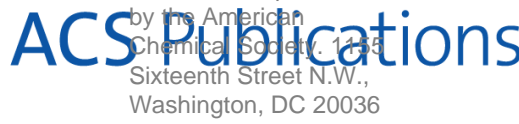

Published by American

Chemical Society.

Copyright () American

Chemical Society.

However, no copyright 


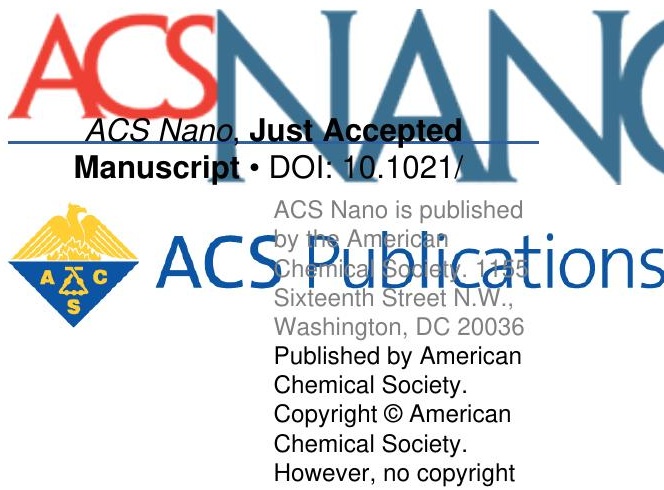




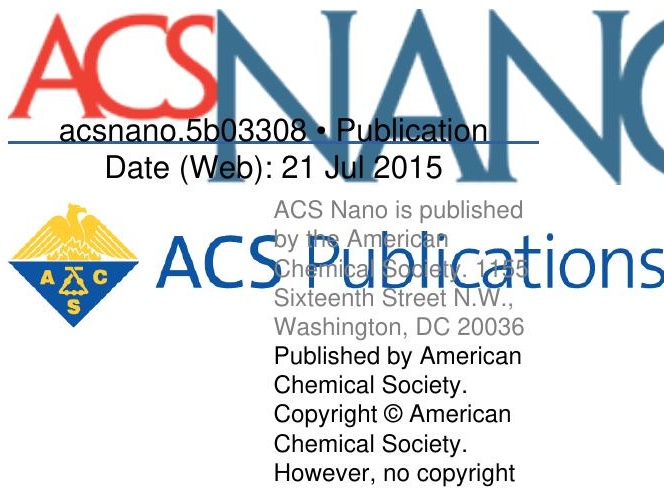




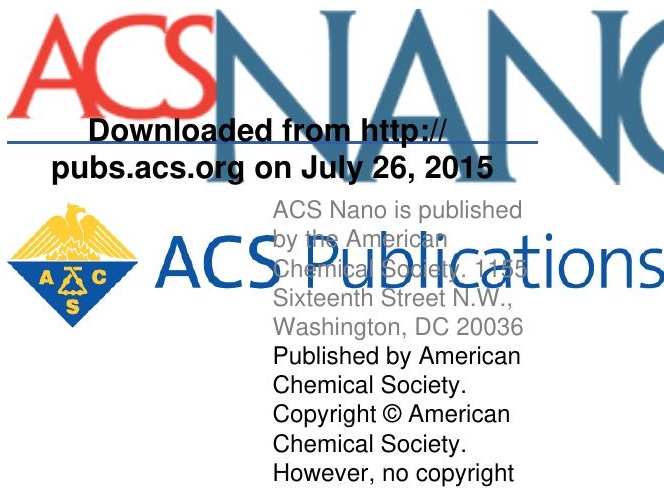



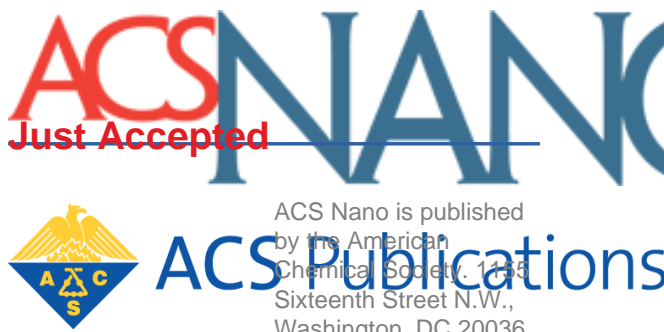

ACS Nano is published

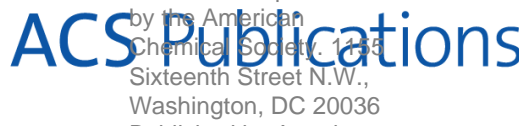

Published by American

Chemical Society.

Copyright (C) American

Chemical Society.

However, no copyright 


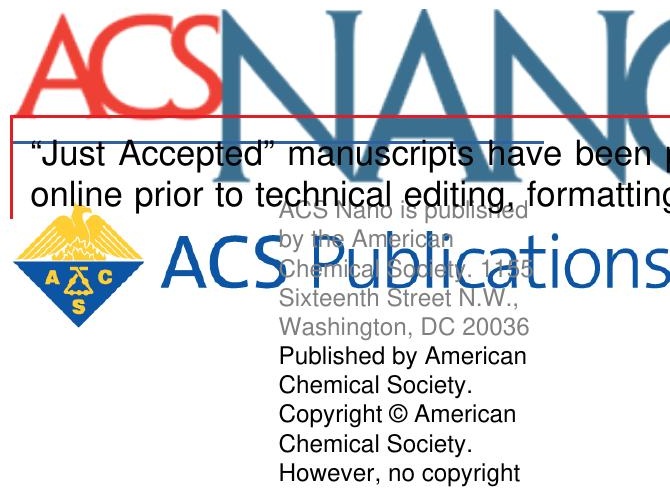


Society provides "Just Accepted" as a dissemination of scientific material as soc appear in full in PDFNformatladecompanied
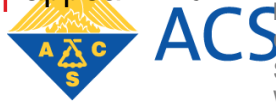

$$
\text { by try Amprictan }
$$

rhomical Sodetr. 1 A

Sixteenth Street N.W.

Washington, DC 20036

Published by American

Chemical Society.

Copyright (C) American

Chemical Society.

However, no copyright 
fully peer reviewed, but should not be con readers and citable by the Digital Object 1 to authors. Thereferen thee ubleist Acceptec
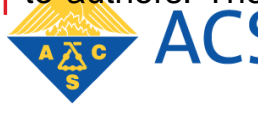

Sy Anmeridan

Sonmina

Sixteenth Street N.W.,

Washington, DC 20036

Published by American

Chemical Society.

Copyright (C) American

Chemical Society.

However, no copyright 


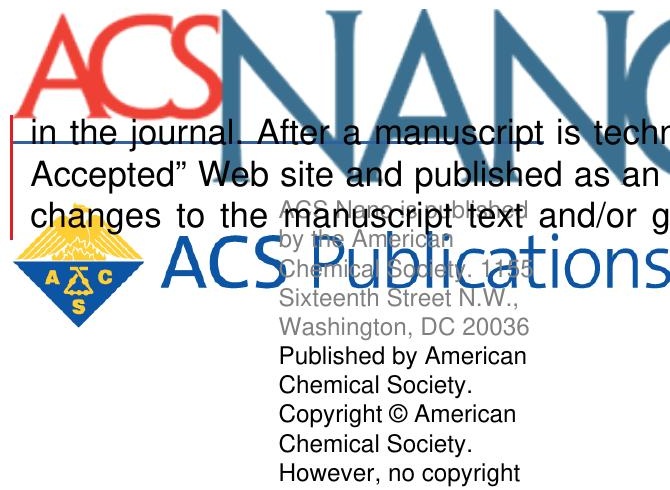


and ethical guidelines that apply to the
or consequences arising from the use of tra

ACS Nano is nublished
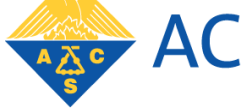

Cixteenth

solde

Washington, DC 20036

Published by American

Chemical Society.

Copyright (c) American

Chemical Society.

However, no copyright 


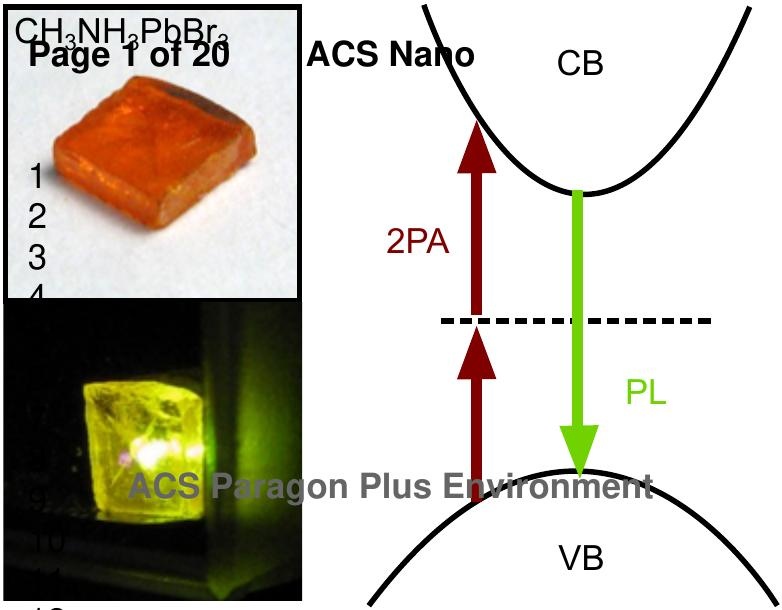

ACs Nało $\quad C B$

2PA

$$
\text { A }
$$$$
\text { - }
$$

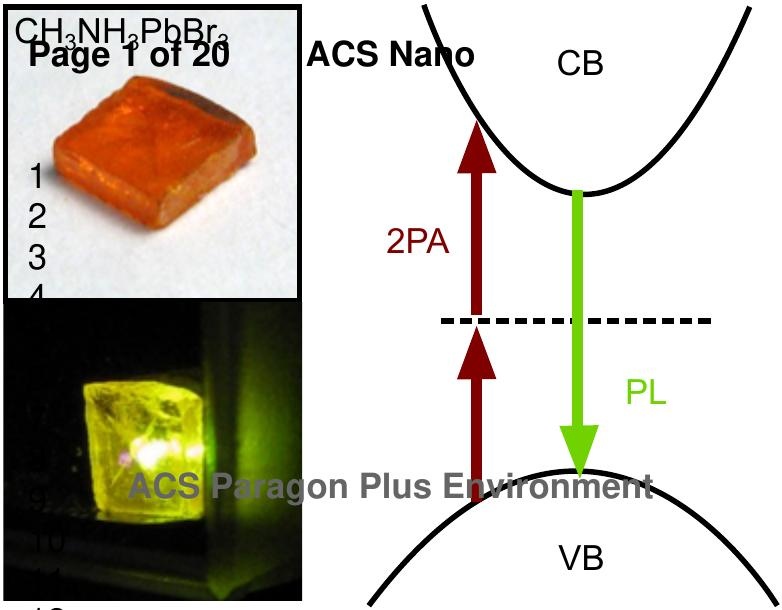

CSP

agon Plus Eyxironment

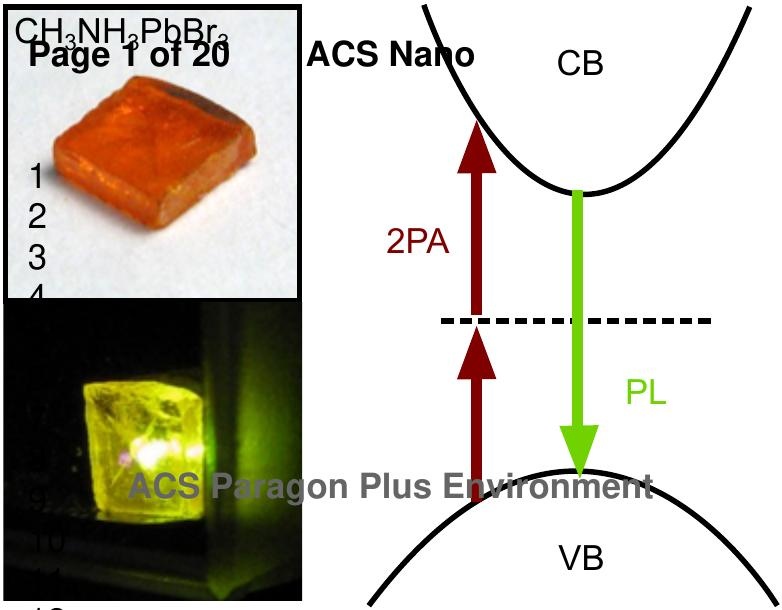




\title{
Two-Photon Absorption in Organometallic Bromide
}

\section{Perovskites}

Grant Walters, ${ }^{1 \dagger}$ Brandon R. Sutherland, ${ }^{1+}$ Sjoerd Hoogland, ${ }^{1}$ Dong Shi, ${ }^{2}$ Riccardo Comin, ${ }^{1}$ Daniel P. Sellan, ${ }^{1}$ Osman M. Bakr, ${ }^{2}$ Edward. H. Sargent ${ }^{1 *}$

${ }^{1}$ Department of Electrical and Computer Engineering, University of Toronto, Toronto, Ontario M5S 3G4, Canada

${ }^{2}$ Division of Physical Sciences and Engineering, Solar and Photovoltaics Engineering Research Center, King Abdullah University of Science and Technology (KAUST), Thuwal 23955-6900, Saudi Arabia

KEYWORDS: perovskite, $\mathrm{CH}_{3} \mathrm{NH}_{3} \mathrm{PbBr}_{3}$, two-photon absorption, photoconductor, autocorrelator

\begin{abstract}
Organometallic trihalide perovskites are solution processed semiconductors that have made great strides in third generation thin film light harvesting and light emitting optoelectronic devices. Recently it has been demonstrated that large, high purity single crystals of these perovskites can be synthesized from the solution phase. These crystals' large dimensions, clean bandgap, and solid-state order, have provided us with a suitable medium to observe and quantify two-photon absorption in perovskites. When $\mathrm{CH}_{3} \mathrm{NH}_{3} \mathrm{PbBr}_{3}$ single crystals
\end{abstract}


are pumped with intense $800 \mathrm{~nm}$ light, we observe band-to-band photoluminescence at $572 \mathrm{~nm}$, indicative of two-photon absorption. We report the nonlinear absorption coefficient of $\mathrm{CH}_{3} \mathrm{NH}_{3} \mathrm{PbBr}_{3}$ perovskites to be $8.6 \mathrm{~cm} \mathrm{GW}^{-1}$ at $800 \mathrm{~nm}$, comparable to epitaxial single crystal semiconductors of similar bandgap. We have leveraged this nonlinear process to electrically autocorrelate a 100 fs pulsed laser using a two-photon perovskite photodetector. This work demonstrates the viability of organometallic trihalide perovskites as a convenient and low-cost nonlinear absorber for applications in ultrafast photonics.

Though formed at low temperature from the solution phase, organometallic halide perovskites possess exceptional material properties. Perovskite thin films exhibit defect densities in the range $10^{16}-10^{17} \mathrm{~cm}^{-3}$, superior to other solution-cast semiconductors processed at similar temperatures. Consistent with the low trap state density are the reported long and balanced diffusion lengths, ${ }^{2}$ large carrier mobilities, ${ }^{3,4}$ and a linear absorption coefficient exceeding $10^{4}$ $\mathrm{cm}^{-1}$ at the band edge. ${ }^{5}$ Driven by these remarkable material properties, perovskite thin film absorbers have demonstrated record solution-processed solar cell power conversion efficiencies up to a certified $20.1 \%{ }^{6}$ Perovskite thin films have also been leveraged in light-emitting devices, demonstrating low-threshold optically pumped lasers ${ }^{1,7,8}$ and high-brightness light-emitting diodes. $^{9}$

Recently, there have been breakthrough demonstrations of the solution-processed growth of large perovskite single crystals. ${ }^{10,11}$ These single crystals offer millimeter-scale dimensions with bulk trap state densities in the range $10^{9}-10^{10} \mathrm{~cm}^{-3}$, comparable to some of the best epitaxial single crystal semiconductors. The impressive energetic landscape in perovskite single crystals has already been functionalized into ultra-low threshold optically pumped lasers. ${ }^{12,13}$ 
We sought to investigate the two-photon absorption in $\mathrm{CH}_{3} \mathrm{NH}_{3} \mathrm{PbBr}_{3}$ perovskites and to demonstrate its potential in low-cost solution processed nonlinear optics. In addition to the importance of understanding the response of organometallic hybrid perovskites under intense radiation, their development as nonlinear optical materials and devices is a new opportunity. ${ }^{14}$ Semiconductor nonlinear absorbers have applications in optical limiting, ${ }^{15}$ characterization of ultrafast optical signals, ${ }^{16}$ lithography, ${ }^{17}$ and microscopy. ${ }^{18}$

Equipped with the newly-discovered process to grow large single crystals, we were able to overcome many existing experimental challenges associated with quantifying two-photon absorption. We hypothesized that the large dimensions of single crystals, as well as their ultraclean bandgaps and long range order would be an ideal material to study perovskite two-photon absorption. The small path length and dominant scattering of conventional perovskite thin films, which proved to limit the accuracy of these measurements, would no longer be an issue. Here we present an accurate quantification of two-photon absorption in $\mathrm{CH}_{3} \mathrm{NH}_{3} \mathrm{PbBr}_{3}$. We demonstrate its applicability in nonlinear optics by autocorrelating a $100 \mathrm{fs}$ laser using a perovskite two photon photodetector. This work is the first report of two-photon absorption and autocorrelation from a solution-processed single crystal organic-inorganic semiconductor.

\section{RESULTS AND DISCUSSION}

To study $\mathrm{CH}_{3} \mathrm{NH}_{3} \mathrm{PbBr}_{3}$ perovskite, we employed the same vapor-assisted single crystal growth technique reported by Shi et al. ${ }^{10}$ Here, the gradual diffusion of an antisolvent into a solution of perovskite precursors promotes seed formation and subsequent crystal growth. The resulting crystals are orange in color with regular rectangular facets (see Figure 1a inset). The large dimensions of the crystals we used enabled accurate measurements of two-photon 
absorption, the magnitude of which is directly impacted by the propagation distance through the sample.

Two-photon absorption is a third-order nonlinear process that becomes relevant at high photon fluences. Two photons, each with energy less than the difference between the material's ground and excited states, are simultaneously absorbed and excite a charge carrier. In semiconductors, this is manifested as an excitation across the bandgap. Two-photon absorption varies with the square of the electric field intensity $I$. The attenuation of light propagating a distance $z$ through a two-photon absorbing medium can thus be written as,

$$
\frac{d I}{d z}=-\alpha I-\beta I^{2}
$$

where $\alpha$ and $\beta$ are the one- (linear) and two-photon absorption coefficients respectively. The solution of this differential equation, expressing the transmission $T$ through a sample of length $L$, is:

$$
T=\frac{I}{I_{0}}=\frac{e^{-\alpha L}}{\beta I_{0} L_{e f f}+1}
$$

where $I_{0}$ is the peak intensity of light entering the sample and $L_{e f f}$ is the effective sample length given by,

$$
L_{e f f}=\frac{1-e^{-\alpha L}}{\alpha}
$$

The intensity of light entering the sample is found by reducing the measured incident intensity by a factor of $(1-R)$ where $R$ is the surface reflectivity. 
In order to achieve degenerate two-photon absorption in our samples we used a high-power mode-locked titanium sapphire laser operating at $800 \mathrm{~nm}$ to provide below-bandgap excitation. The bandgap of $\mathrm{CH}_{3} \mathrm{NH}_{3} \mathrm{PbBr}_{3}$ is $2.21 \mathrm{eV},{ }^{10}$ with an absorption onset at $560 \mathrm{~nm}$ as shown in Figure 1a. This onset is sharp, leading to nearly constant absorption for above-bandgap radiation. The step-like behavior in the absorption ensured the samples were free from linear absorption of the incident laser light. Previous transient absorption measurements on $\mathrm{CH}_{3} \mathrm{NH}_{3} \mathrm{PbBr}_{3}$ single crystals using above-bandgap pump and below-bandgap probe lasers have shown significant changes in optical absorption within picoseconds of excitation; however, the excited population that allows for this change in absorption quickly decays such that the material regains its belowbandgap transparency within nanoseconds. ${ }^{10}$ We have used ultra-short pulses (100 fs) with a periodicity of $13 \mathrm{~ns}$ in our experiments. The use of ultra-short pulses in our experiments and the absence of linear absorption means photoinduced absorption associated with excited-state carriers was negligible and did not affect the accuracy of our measurements.

Strikingly bright green light is visibly emitted when the samples are placed in the path of the laser (Figure 1a inset). This photoluminescence was direct evidence that our samples were in-fact absorbing the below-bandgap $800 \mathrm{~nm}$ radiation through two-photon absorption. The resulting emission spectrum in Figure 1a shows a sharp and narrow peak at $572 \mathrm{~nm}$-with a wavelength dependence unchanged from linearly induced photoluminescence. Thus the perovskite crystals behave as an effective upconverter for near infrared radiation. A simple schematic of the twophoton absorption and induced photoluminescence process is given in Figure $1 \mathrm{~b}$.

The $\mathrm{z}$-scan procedure ${ }^{19}$ is the most widely-used method for measuring two-photon absorption coefficients because of its simplicity and ease of data interpretation. It involves translating the sample along the path of a focused laser beam in order to vary the incident intensity and find $\beta$. 
Although convenient, the method can be prone to artefacts and overestimation with less-thanideal samples. To avoid possible changes in scattering associated with changes in the spot-size as the sample is translated for z-scans, we opted to alternatively measure the two-photon absorption coefficient for $\mathrm{CH}_{3} \mathrm{NH}_{3} \mathrm{PbBr}_{3}$ through static intensity-dependent transmission measurements. This straight-forward technique provides an accurate description of two-photon absorption behavior. $^{20}$ These measurements were made by placing the sample at the waist of the focused laser beam, varying the laser intensity with a filter wheel, and monitoring incident and transmitted power.

A typical plot of inverse transmission versus peak intensity is given in Figure 2a. The plot shows the requisite linear increase with intensity according to equation 2 accompanied by an expected slight downward curvature, the latter behavior indicating temporal and spatial broadening of the beam towards the rear of the sample when subjected to high intensities. ${ }^{20}$ The solid line is a least-squares fit according to the equations for temporally and spatially Gaussian pulses developed by Sheik-Bahae et al. ${ }^{19}$ :

$$
\begin{gathered}
T\left(I_{0}\right)=\sum_{m=0}^{\infty} \frac{\left[-q\left(I_{0}\right)\right]^{m}}{(m+1)^{\frac{3}{2}}}, \\
q\left(I_{0}\right)=\beta I_{0} L_{e f f},
\end{gathered}
$$

The sum in equation 4 was performed for 20 terms to ensure proper convergence of the function. Although linear absorption can be ignored for these samples, they often showed significant scattering and so this loss mechanism was treated as linear absorption in accordance with Ref. ${ }^{20}$. Repeated measurements on several samples yielded an average absorption coefficient of $8.6 \pm$ $0.5 \mathrm{~cm} \mathrm{GW}^{-1}$ (error indicates one standard deviation). For comparison, $\mathrm{CdS}\left(\mathrm{E}_{\mathrm{g}}=2.42 \mathrm{eV}\right)$ and 
$\operatorname{CdSe}\left(E_{\mathrm{g}}=1.74 \mathrm{eV}\right)$ have absorption coefficients of $5.5 \mathrm{~cm} \mathrm{GW}^{-1}($ at $530 \mathrm{~nm})$ and $18 \mathrm{~cm} \mathrm{GW}^{-1}$ (at $1060 \mathrm{~nm}$ ) respectively. ${ }^{21}$ The dependence of two-photon absorption with band-gap for semiconductors is well established and we find our value for $\mathrm{CH}_{3} \mathrm{NH}_{3} \mathrm{PbBr}_{3}$ agrees well with the reported scaling laws. ${ }^{20-22}$ The experimental value has only a $14 \%$ error with our own prediction based on these relations: $10 \mathrm{~cm} \mathrm{GW}^{-1}$. Consideration of the corresponding scaling laws for the nonlinear refractive index and the Kramers-Kronig relation between the two-photon absorption coefficient and the nonlinear refractive index shows that the nonlinear reflection due to changes in the material's refractive index (a maximum change of $10^{-5}$ ) is negligible and does not impact the measured two-photon absorption coefficient. ${ }^{22}$

The use of single crystals enabled us to measure the polarization dependence of two-photon absorption in the perovskites. These measurements are fundamentally impossible with polycrystalline thin films due to their random lattice orientations. Figure $2 \mathrm{~b}$ shows the polarization dependence of two-photon absorption for $\mathrm{CH}_{3} \mathrm{NH}_{3} \mathrm{PbBr}_{3}$. Plotted is the transmission scaled by the incident intensity $T / I_{I n}-$ a quantity directly proportional to the absorption coefficient. A half-wave plate was used to rotate the incident beam's electric field polarization an angle $\theta$ relative to the [100] crystallographic axis and the incident propagation along the [001] direction such that the polarization rotated through the crystallographic xy plane. $\mathrm{CH}_{3} \mathrm{NH}_{3} \mathrm{PbBr}_{3}$ is cubic with the space group $P m 3 m$ at room temperature, ${ }^{10}$ and so the polarization dependence can be modeled with:

$$
\frac{T}{I_{I n}}=A\left[1+2 \sigma\left[\sin ^{4}(\theta+\varphi)-\sin ^{2}(\theta+\varphi)\right]\right]
$$


where $A$ is related to the imaginary component of the susceptibility tensor element $\chi_{x x x x}^{(3)}, \sigma$ is the sample anisotropy parameter, and $\varphi$ is the wave-plate angular offset. ${ }^{23-25}$ A least-squares fit is shown in Figure $2 \mathrm{~b}$ and yields an anisotropy parameter of $\sigma=-0.07$.

$\mathrm{CH}_{3} \mathrm{NH}_{3} \mathrm{PbBr}_{3}$ and other organometallic perovskites have previously been predicted, through density functional theory calculations, to exhibit a large spin-orbit coupling due to the heavy lead atoms. $^{26-31}$ This coupling splits the degenerate conduction band, such that a doublet band is lowered and a quartet band is raised in energy. ${ }^{26}$ These predictions are supported by an agreement between the experimentally measured and the predicted values for the bandgap. For semiconductors in general, the valence band to lowest conduction band transitions do not provide any orientational dependence for two-photon absorption. ${ }^{24,25,32}$ Anisotropic behavior may be introduced either from direct transitions between the valence bands and the higher conduction bands or from state mixing between these bands. ${ }^{24,25,32}$ These mechanisms are minimal in $\mathrm{CH}_{3} \mathrm{NH}_{3} \mathrm{PbBr}_{3}$ where the conduction bands have a large energetic separation, consistent with the small anisotropy parameter observed.

We utilized the nonlinear properties of our perovskite single crystals for autocorrelation purposes. This can be done with measurements of the nonlinear response of transmission, photoluminescence intensity, second harmonic generation, or electrical current. In line with the facile processing of the perovskite crystals and their exceptional electrical properties we aimed to use electrical current, one of the most straightforward signals to measure, as our autocorrelation signal.

We fabricated a simple photoconductor by depositing silver contacts on opposite ends of a single crystal. A schematic of the photodetector, along with the crystal dimensions is shown in 
Figure 3a. The sample is illuminated from the side, resulting in single-pass two-photon absorption of $800 \mathrm{~nm}$ light. The dark current-voltage characteristic of this crystal is shown in Figure 3b. This crystal has an electrical conductivity of $10^{-5} \Omega^{-1} \mathrm{~cm}^{-1}$.

The photocurrent (light minus dark current) generated as a result of single-pass absorption of $800 \mathrm{~nm}$ light is given as a function of peak intensity of the pump laser at a fixed bias of $50 \mathrm{~V}$ in Figure 3c. We tested control samples of just glass and just silver and no photocurrent is observed. Photoconductors based on linear absorption have a photocurrent which increases sublinearly (power-law dependence $\mathrm{n}<1$ ) with input intensity (see Supporting Information Figure S1a). ${ }^{33}$ However, the two-photon absorption probability increases with the square of input intensity $(n=2)$. We therefore expect a photocurrent dependence on input intensity which is less than $n=2$, but greater than linear photoconduction $(n<1)$, which is what we observe.

The responsivity (efficiency in $\mathrm{A} \mathrm{W}^{-1}$ ) of the two photon photodetector at a fixed bias of $50 \mathrm{~V}$ as a function of input intensity is given in Figure $3 \mathrm{~d}$. The responsivity is of order $10^{-7}$. This value is five orders of magnitude less than the upper limit in the absence of photoconductive gain as determined by the two-photon absorption coefficient. Low responsivity is expected given that perovskites, with their low trap state density and balanced carrier mobilities, exhibit low intrinsic photoconductive gain. ${ }^{34}$ Despite the low gain, due to the large number of total absorbed photons, the device output photocurrent is in the range $10 \mathrm{nA}-1 \mu \mathrm{A}$, readily measurable using a conventional current meter. The responsivity increases $3.5 x$ over 1 decade of input intensity. This is contrary to linear photoconductors, which have a responsivity that decreases with increasing input intensity (see Supporting Information Figure S1b). This upward trend demonstrates that the photocurrent is a result of nonlinear absorption and not linear processes such as thermal generation or trap-mediated absorption. 
A schematic of our perovskite autocorrelator is shown in Figure 4a. A 100 fs, $800 \mathrm{~nm}$ pulse passes through a 50/50 beam splitter (BS). One arm is sent to a retro-reflector on a delay line. The two arms meet again at a second 50/50 splitter which sends half of the total light to a power meter (det), the other half through a focusing lens onto the two-photon absorbing perovskite photodetector. The generated photocurrent is collected using a current meter and monitored as a function of the delay line position. The resulting current-time trace is shown in Figure 4b. Our autocorrelator configuration is a modified Mach-Zehnder interferometer where the two pulses have the same phase and polarization. With this configuration, we observe constructive and destructive interference fringes corresponding to the overlap of the time-varying electrical fields. The envelope of these fringes corresponds to the intensity overlap of the pulses in time. We fit the top and bottom envelopes to a $\operatorname{sech}^{2}(\mathrm{t})$ function, shown in red. The resulting autocorrelation has a full-width at half-maximum (FWHM) of 160 fs. Since we are autocorrelating the electric field, but we wish to know the intensity profile, there is a deconvolution factor to be applied. For $\operatorname{a~sech}^{2}(\mathrm{t})$ input pulse intensity profile, the deconvolution factor is $0.65,{ }^{35}$ resulting in a measured pulse duration of $104 \mathrm{fs}$. This is in excellent agreement with the $100 \mathrm{fs}$ specification of our laser. The fringe spacing, limited by the resolution of our motorized stage, is approximately every $3 / 2$ of a wavelength, shown in Figure 4c.

\section{CONCLUSION}

This work demonstrates the prospects of perovskites as solution processed two-photon absorbers for applications in nonlinear optics. With just readily available chemical precursors, researchers can fabricate large single crystal two-photon absorbers to accurately and conveniently autocorrelate ultrafast lasers. Yet, the nonlinear optical properties of perovskites remain largely unexplored. Future work will focus on investigating new avenues for perovskites 
as nonlinear optical materials, such as studying secondary harmonic generation, optical limiting, and self-focusing.

\section{METHODS}

$\mathrm{CH}_{3} \mathrm{NH}_{3} \mathrm{PbBr}_{3}$ single crystals were prepared using a vapor-assisted crystallization procedure as previously reported. ${ }^{10}$ In brief, the vapor from an antisolvent (dichloromethane, in our case) was allowed to slowly diffuse into a solution of perovskite precursors, lead bromide $\left(\mathrm{PbBr}_{2}\right)$ and methylammonium bromide $\left(\mathrm{CH}_{3} \mathrm{NH}_{3} \mathrm{Br}\right)$ in $\mathrm{N}, \mathrm{N}$-dimethylformamide, such that precursors spontaneously nucleated and co-crystallized in a self-sustained process. Crystals were kept in the mother liquor until use in optical experiments. Their dimensions were measured using a Mitutoyo digital caliper to within $10 \mu \mathrm{m}$.

Linear optical absorption was characterized using a PerkinElmer Lambda 950 UV/Vis/NIR spectrophotometer in the wavelength range $250-900 \mathrm{~nm}$ and using $2 \mathrm{~nm}$ incremental steps. Photoluminescence induced by linear absorption was measured using a Liconix Helium Cadmium 442 nm excitation laser and Ocean Optics USB2000 spectrometer.

For all nonlinear absorption experiments, a mode-locked Mira 900-F Ti:Sapphire laser was used as the excitation source. The pulse duration was $100 \mathrm{fs}$ (FWHM) at a repetition rate of 76 MHz. The laser was tuned to an output wavelength of $800 \mathrm{~nm}$ and produced horizontally polarized $\mathrm{TEM}_{00}$-mode single pulses. Power measurements were made with an Ophir LaserStar Dual Channel power and energy meter with photodiode sensors which had an accuracy of $\pm 5 \%$ after attenuation at $800 \mathrm{~nm}$. 
Two-photon absorption coefficients were measured by placing the sample at the focus of a lens with a $15 \mathrm{~cm}$ focal length and by attenuating the incident laser radiation with a neutral density filter wheel. The incident and transmitted light was measured with power meters placed before and after the sample. The lens focused the beam to a spot size of $35 \mu \mathrm{m}$ (half-width at $1 / \mathrm{e}^{2}$ ) as determined with a CCD camera setup. For most measurements, the incident beam was chopped at $400 \mathrm{~Hz}$ in order to prevent sample heating and optical damage. To measure the polarization dependence of two-photon absorption, a rotating half-wave plate was placed in the path of the incident beam. Predicted absorption coefficients were determined through the scaling law,

$$
\beta=3100 \frac{\sqrt{E_{P}}}{n^{2} E_{g}^{3}} \frac{\left(2 \hbar \omega / E_{g}-1\right)^{3 / 2}}{\left(2 \hbar \omega / E_{g}\right)^{5}},
$$

where,

$$
E_{P}=\frac{2 P^{2} m}{\hbar^{2}}(\text { in } \mathrm{eV})
$$

and $E_{g}$ is the bandgap (eV), $\hbar \omega$ is the field energy $(\mathrm{eV}), m$ is the electron mass, $n$ is the linear refractive index (assumed 2.5) and $P$ is the Kane momentum parameter (assumed $4.5 \times 10^{-27} \mathrm{~J}$ $\mathrm{m})^{20-22}$

Photoconduction measurements were done using a device made from a single crystal with wires bonded to opposing crystal faces with conductive silver paste. Two-photon absorption photocurrent was measured using a Keithley 2410 High Voltage Source Meter (also used as a bias source).

For autocorrelation experiments the incident laser beam was split into two branches, such that a motorized translation stage with a retroreflector could be added to introduce a pulse-delay for 
one of the paths. The separate paths were then rerouted into a single collinear path that was incident on our sample. Photocurrent was measured as a function of the retroreflector position in order to detect pulse-overlap and determine the autocorrelation width.

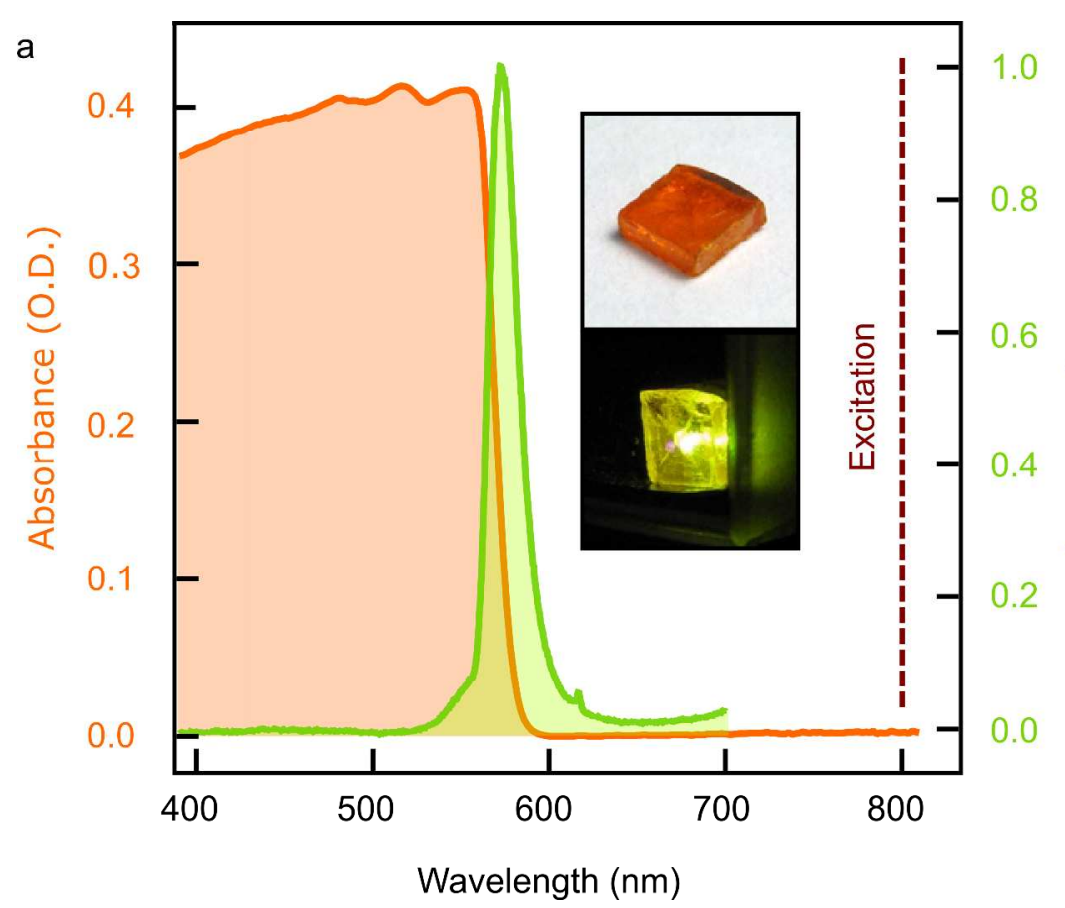

b

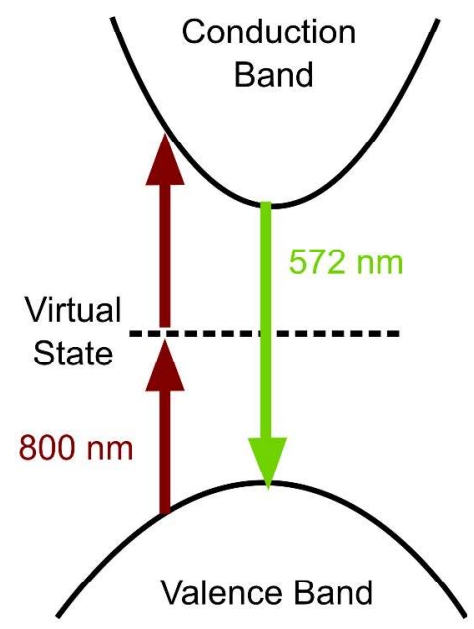

Figure 1. Optical properties. a) Linear optical absorbance (left axis) and normalized two-photoninduced photoluminescence (TPPL, right axis) as a function of wavelength for a single $\mathrm{CH}_{3} \mathrm{NH}_{3} \mathrm{PbBr}_{3}$ crystal. Photoluminescence peaks at $572 \mathrm{~nm}$. Insets show the crystal (dimensions $\sim 1 \times 5 \times 5 \mathrm{~mm}$ ) under room light (upper) and brightly luminescing when exposed to intense 800 nm laser radiation (lower). The visibly observed photoluminescence is a direct signature of twophoton absorption. b) Schematic showing two-photon absorption of $800 \mathrm{~nm}$ light and upconversion to $572 \mathrm{~nm}$ photoluminescence. 
a

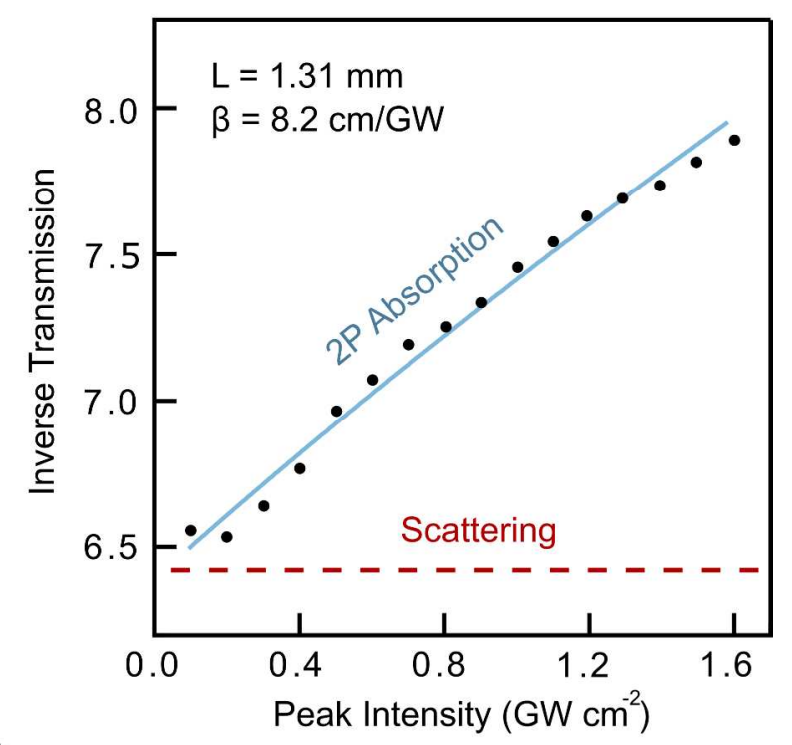

b

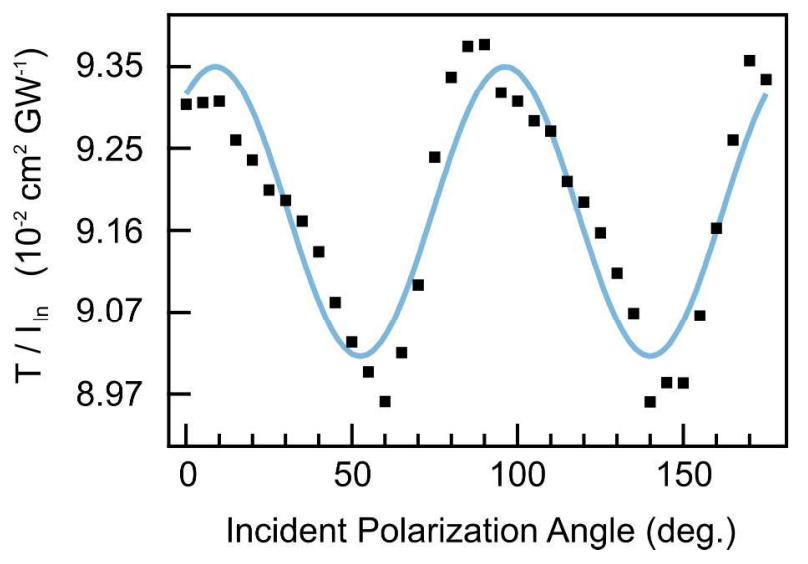

Figure 2. Two-photon absorption coefficient. a) Inverse transmission versus peak intensity for a typical single $\mathrm{CH}_{3} \mathrm{NH}_{3} \mathrm{PbBr}_{3}$ crystal. The solid line is a least-squares fit to the data points using equations 4-5 resulting in a two-photon absorption coefficient of $8.2 \mathrm{GW} \mathrm{cm}^{-1}$. Dashed red line indicates linear loss, primarily due to scattering. b) Transmission scaled by incident intensity versus electric field polarization angle relative [100] crystallographic axis (offset by 8 degrees). Solid line is a least-squares fit to equation 6 and yields an anisotropy parameter of -0.07 . 
a

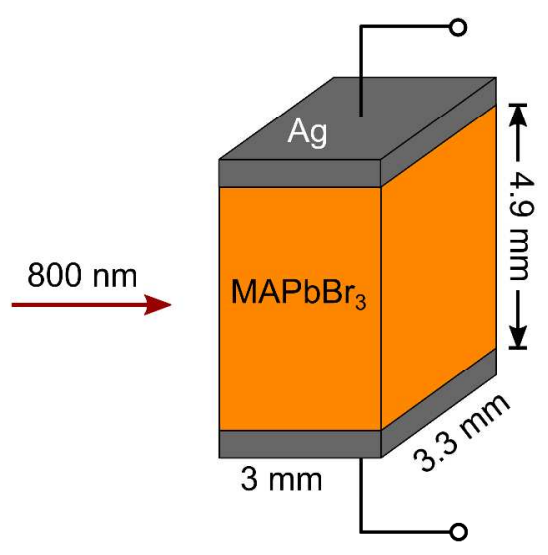

c

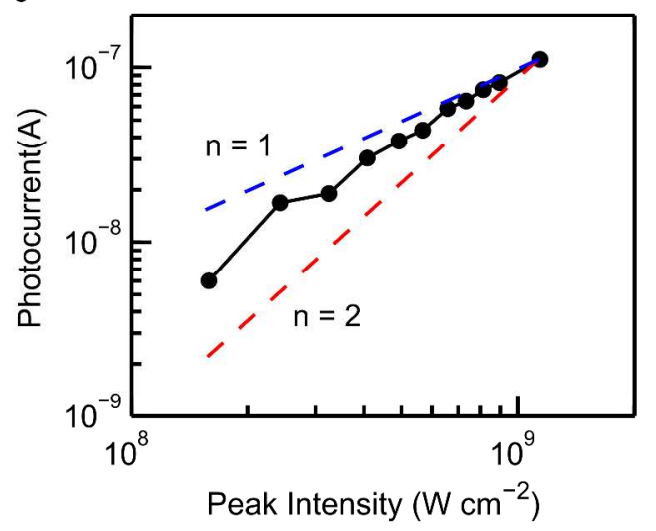

b

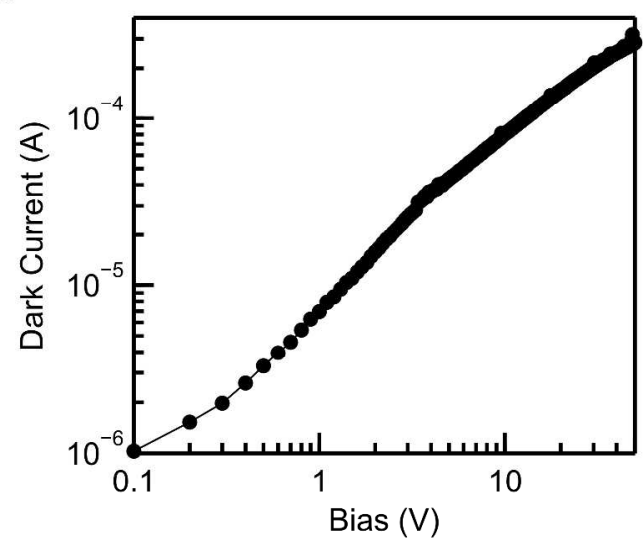

d

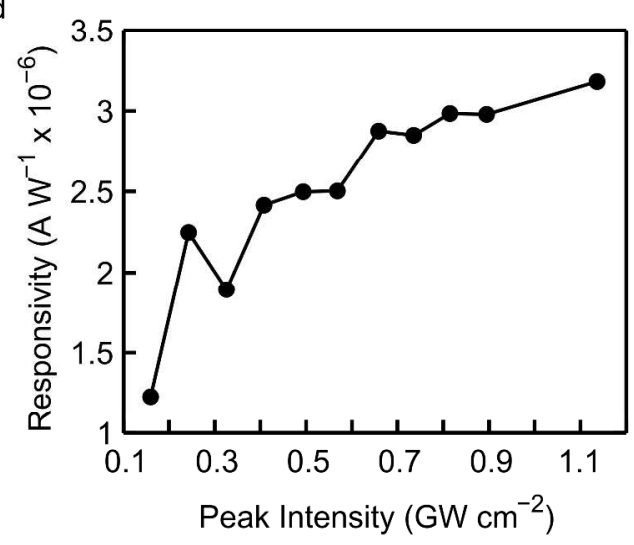

Figure 3. $\mathrm{CH}_{3} \mathrm{NH}_{3} \mathrm{PbBr}_{3}$ two-photon photodetector. a) Device schematic. b) Dark currentvoltage characteristic. The perovskite photoconductor has an electrical conductivity of order $10^{-5}$ $\Omega^{-1} \mathrm{~cm}^{-1}$.c) Net photocurrent (light current minus dark current) dependence on input light intensity at a bias of $50 \mathrm{~V}$. The photodetector exhibits an intensity dependence which is a combination of two competing trends, the square $(n=2)$ dependence of two-photon absorption, and the sublinear $(\mathrm{n}<1)$ dependence of photoconductive gain. d) Resistivity (photocurrent / input power) dependence on input intensity at a bias of $50 \mathrm{~V}$. Contrary to linear photoconductors, the responsivity of the perovskite two-photon photoconductor does not decrease with increasing input intensity due to the squared dependence of absorption. 
a

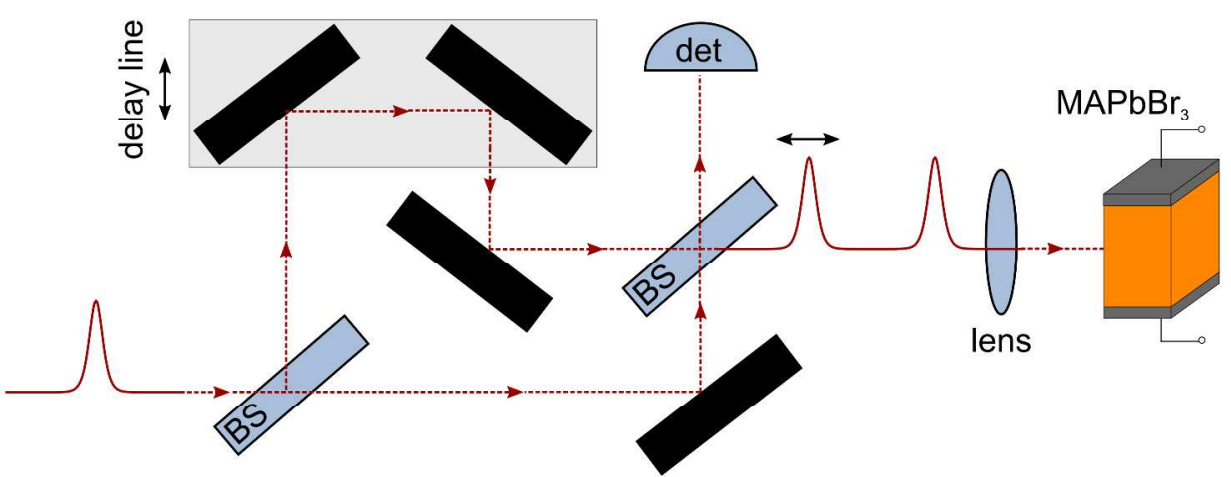

b

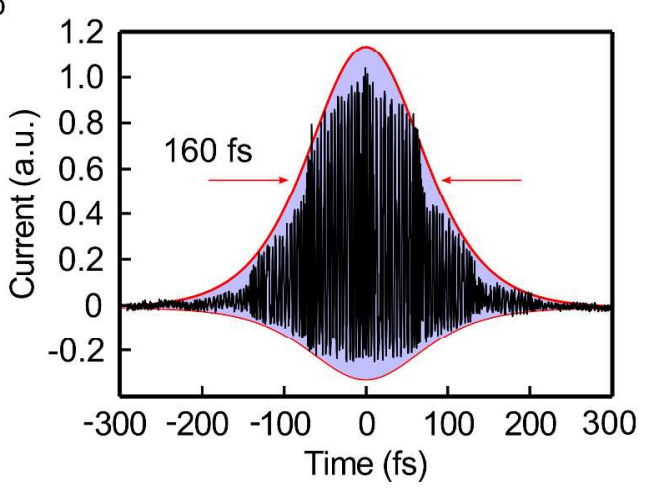

C

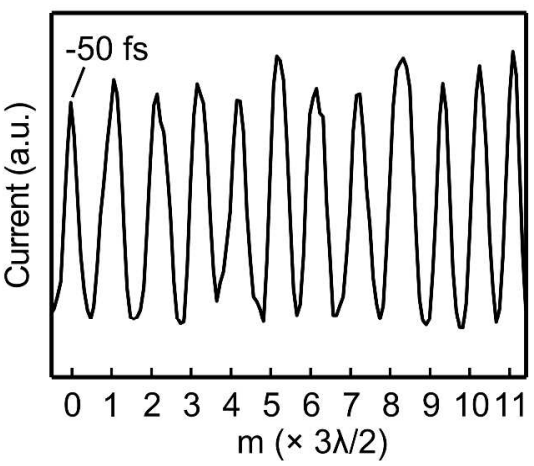

Figure 4. Autocorrelation of a $100 \mathrm{fs}$ Ti:Sapphire laser using a $\mathrm{CH}_{3} \mathrm{NH}_{3} \mathrm{PbBr}_{3}$ two-photon detector. a) Optical schematic. The input laser pulse is split into two, with one pulse variably delayed in time using a retroreflector on a motorized linear translation stage. Photocurrent generated by the two-photon perovskite photodetector is used to autocorrelate the beam pulse. b) Autocorrelation signal. We observe a $160 \mathrm{fs}$ FWHM in the autocorrelation envelope, corresponding to a $100 \mathrm{fs}$ pulse width. c) Electric field interference. We resolve constructive interference approximately every $3 \lambda / 2$. 


\section{ASSOCIATED CONTENT}

Supporting Information Available: Photoconductor operating under above-bandgap

illumination. This material is available free of charge via the Internet at http://pubs.acs.org.

\section{AUTHOR INFORMATION \\ Corresponding Author \\ * Email: ted.sargent@utoronto.ca}

\section{Author Contributions}

The manuscript was written through contributions of all authors. All authors have given approval to the final version of the manuscript. $†$ These authors contributed equally.

\section{ACKNOWLEDGMENT}

This publication is based in part on work supported by Award KUS-11-009-21, made by King Abdullah University of Science and Technology (KAUST), by the Ontario Research Fund Research Excellence Program, and by the Natural Sciences and Engineering Research Council (NSERC) of Canada.

\section{REFERENCES}

(1) Xing, G.; Mathews, N.; Lim, S. S.; Yantara, N.; Liu, X.; Sabba, D.; Grätzel, M.; Mhaisalkar, S.; Sum, T. C. Low-Temperature Solution-Processed Wavelength-Tunable Perovskites for Lasing. Nat. Mater. 2014, 13, 476-480.

(2) Xing, G.; Mathews, N.; Sun, S.; Lim, S. S.; Lam, Y. M.; Grätzel, M.; Mhaisalkar, S.; Sum, T. C. Long-Range Balanced Electron-and Hole-Transport Lengths in Organic-Inorganic CH3NH3PbI3. Science 2013, 342, 344-347.

(3) Stoumpos, C. C.; Malliakas, C. D.; Kanatzidis, M. G. Semiconducting Tin and Lead Iodide Perovskites with Organic Cations: Phase Transitions, High Mobilities, and NearInfrared Photoluminescent Properties. Inorg. Chem. 2013, 52, 9019-9038. 
(4) Shao, Y.; Xiao, Z.; Bi, C.; Yuan, Y.; Huang, J. Origin and Elimination of Photocurrent Hysteresis by Fullerene Passivation in CH3NH3PbI3 Planar Heterojunction Solar Cells. Nat. Commun. 2014, 5, 5784.

(5) Green, M. A.; Ho-Baillie, A.; Snaith, H. J. The Emergence of Perovskite Solar Cells. Nat. Photonics 2014, 8, 506-514.

(6) Yang, W. S.; Noh, J. H.; Jeon, N. J.; Kim, Y. C.; Ryu, S.; Seo, J.; Seok, S. I. HighPerformance Photovoltaicperovskite Layers Fabricated Throughintramolecular Exchange. Science 2015, 348, 1234-1237.

(7) Deschler, F.; Price, M.; Pathak, S.; Klintberg, L. E.; Jarausch, D.-D.; Higler, R.; Hüttner, S.; Leijtens, T.; Stranks, S. D.; Snaith, H. J.; et al. High Photoluminescence Efficiency and Optically Pumped Lasing in Solution-Processed Mixed Halide Perovskite Semiconductors. J. Phys. Chem. Lett. 2014, 5, 1421-1426.

(8) Sutherland, B. R.; Hoogland, S.; Adachi, M. M.; Wong, C. T. O.; Sargent, E. H. Conformal Organohalide Perovskites Enable Lasing on Spherical Resonators. ACS Nano 2014, 8, 10947-10952.

(9) Tan, Z.-K.; Moghaddam, R. S.; Lai, M. L.; Docampo, P.; Higler, R.; Deschler, F.; Price, M.; Sadhanala, A.; Pazos, L. M.; Credgington, D.; et al. Bright Light-Emitting Diodes Based on Organometal Halide Perovskite. Nat. Nanotechnol. 2014, 9, 687-692.

(10) Shi, D.; Adinolfi, V.; Comin, R.; Yuan, M.; Alarousu, E.; Buin, A.; Chen, Y.; Hoogland, S.; Rothenberger, A.; Katsiev, K.; et al. Low Trap-State Density and Long Carrier Diffusion in Organolead Trihalide Perovskite Single Crystals. Science 2015, 347, 519522.

(11) Dong, Q.; Fang, Y.; Shao, Y.; Mulligan, P.; Qiu, J.; Cao, L.; Huang, J. Electron-Hole Diffusion Lengths $>175 \mathrm{Mm}$ in Solution-Grown CH3NH3PbI3 Single Crystals. Science 2015, 347, 967-970.

(12) Zhu, H.; Fu, Y.; Meng, F.; Wu, X.; Gong, Z.; Ding, Q.; Gustafsson, M. V.; Trinh, M. T.; Jin, S.; Zhu, X.-Y. Lead Halide Perovskite Nanowire Lasers with Low Lasing Thresholds and High Quality Factors. Nat. Mater. 2015, DOI: 10.1038/nmat4271.

(13) Liao, Q.; Hu, K.; Zhang, H.; Wang, X.; Yao, J.; Fu, H. Perovskite Microdisk Microlasers Self-Assembled from Solution. Adv. Mater. 2015, DOI: 10.1002/adma.201500449.

(14) Stoumpos, C. C.; Frazer, L.; Clark, D. J.; Kim, Y. S.; Rhim, S. H.; Freeman, A. J.; Ketterson, J. B.; Jang, J. I.; Kanatzidis, M. G. Hybrid Germanium Iodide Perovskite Semiconductors: Active Lone Pairs, Structural Distortions, Direct and Indirect Energy Gaps, and Strong Nonlinear Optical Properties. J. Am. Chem. Soc. 2015, 137, 6804-6819.

(15) Liberman, V.; Rothschild, M.; Bakr, O. M.; Stellacci, F. Optical Limiting with Complex Plasmonic Nanoparticles. J. Opt. 2010, 12, 065001.

(16) Chong, E. Z.; Watson, T. F.; Festy, F. Autocorrelation Measurement of Femtosecond Laser Pulses Based on Two-Photon Absorption in GaP Photodiode. Appl. Phys. Lett. 2014, 105, 062111.

(17) Haske, W.; Chen, V. W.; Hales, J. M.; Dong, W.; Barlow, S.; Marder, S. R.; Perry, J. W. $65 \mathrm{Nm}$ Feature Sizes Using Visible Wavelength 3-D Multiphoton Lithography. Opt. Express 2007, 15, 3426-3436.

(18) Zipfel, W. R.; Williams, R. M.; Webb, W. W. Nonlinear Magic: Multiphoton Microscopy in the Biosciences. Nat. Biotechnol. 2003, 21, 1369-1377. 
(19) Sheik-Bahae, M.; Said, A. A.; Wei, T.-H.; Hagan, D. J.; Van Stryland, E. W. Sensitive Measurement of Optical Nonlinearities Using a Single Beam. Quantum Electron. IEEE J. Of 1990, 26, 760-769.

(20) Van Stryland, E. W.; Vanherzeele, H.; Woodall, M. A.; Soileau, M. J.; Smirl, A. L.; Guha, S.; Boggess, T. F. Two Photon Absorption, Nonlinear Refraction, and Optical Limiting in Semiconductors. Opt. Eng. 1985, 24, 613-623.

(21) Van Stryland, E. W.; Woodall, M. A.; Vanherzeele, H.; Soileau, M. J. Energy Band-Gap Dependence of Two-Photon Absorption. Opt. Lett. 1985, 10, 490-492.

(22) Sheik-Bahae, M.; Hagan, D. J.; Van Stryland, E. W. Dispersion and Band-Gap Scaling of the Electronic Kerr Effect in Solids Associated with Two-Photon Absorption. Phys. Rev. Lett. 1990, 65, 96.

(23) DeSalvo, R.; Sheik-Bahae, M.; Said, A. A.; Hagan, D. J.; Van Stryland, E. W. Z-Scan Measurements of the Anisotropy of Nonlinear Refraction and Absorption in Crystals. Opt. Lett. 1993, 18, 194-196.

(24) Dvorak, M. D.; Schroeder, W. A.; Andersen, D. R.; Smirl, A. L.; Wherrett, B. S. Measurement of the Anisotropy of Two-Photon Absorption Coefficients in Zincblende Semiconductors. Quantum Electron. IEEE J. Of 1994, 30, 256-268.

(25) Hutchings, D. C.; Wherrett, B. S. Theory of Anisotropy of Two-Photon Absorption in Zinc-Blende Semiconductors. Phys. Rev. B 1994, 49, 2418.

(26) Jishi, R. A.; Ta, O. B.; Sharif, A. A. Modeling of Lead Halide Perovskites for Photovoltaic Applications. J. Phys. Chem. C 2014, 118, 28344-28349.

(27) Brivio, F.; Butler, K. T.; Walsh, A.; van Schilfgaarde, M. Relativistic Quasiparticle SelfConsistent Electronic Structure of Hybrid Halide Perovskite Photovoltaic Absorbers. Phys. Rev. B 2014, 89 .

(28) Giorgi, G.; Fujisawa, J.-I.; Segawa, H.; Yamashita, K. Small Photocarrier Effective Masses Featuring Ambipolar Transport in Methylammonium Lead Iodide Perovskite: A Density Functional Analysis. J. Phys. Chem. Lett. 2013, 4, 4213-4216.

(29) Even, J.; Pedesseau, L.; Jancu, J.-M.; Katan, C. Importance of Spin-Orbit Coupling in Hybrid Organic/Inorganic Perovskites for Photovoltaic Applications. J Phys Chem Lett 2013, 4, 2999-3005.

(30) Mosconi, E.; Amat, A.; Nazeeruddin, M. K.; Grätzel, M.; De Angelis, F. First-Principles Modeling of Mixed Halide Organometal Perovskites for Photovoltaic Applications. J. Phys. Chem. C 2013, 117, 13902-13913.

(31) Pedesseau, L.; Jancu, J.-M.; Rolland, A.; Deleporte, E.; Katan, C.; Even, J. Electronic Properties of 2D and 3D Hybrid Organic/inorganic Perovskites for Optoelectronic and Photovoltaic Applications. Opt. Quantum Electron. 2014, 46, 1225-1232.

(32) Hutchings, D. C.; Wherrett, B. S. Theory of the Polarization Dependence of Two-Photon Absorption in Zinc-Blende Semiconductors. J. Mod. Opt. 1994, 41, 1141-1149.

(33) Konstantatos, G.; Clifford, J.; Levina, L.; Sargent, E. H. Sensitive Solution-Processed Visible-Wavelength Photodetectors. Nat. Photonics 2007, 1, 531-534.

(34) Hu, X.; Zhang, X.; Liang, L.; Bao, J.; Li, S.; Yang, W.; Xie, Y. High-Performance Flexible Broadband Photodetector Based on Organolead Halide Perovskite. Adv. Funct. Mater. 2014, 24, 7373-7380.

(35) Miller, A.; Reid, D. T. Ultrafast Photonics; Scottish Graduate Series; CRC Press, 2004. 